\title{
Investigation of Sound Speed Errors in Adaptive Beamforming
}

\author{
Holfort, Iben Kraglund; Gran, Fredrik; Jensen, Jørgen Arendt
}

Published in:

Proceedings of the IEEE International Ultrasonics Symposium

Link to article, DOI:

10.1109/ULTSYM.2008.0260

Publication date:

2008

Document Version

Publisher's PDF, also known as Version of record

Link back to DTU Orbit

Citation (APA):

Holfort, I. K., Gran, F., \& Jensen, J. A. (2008). Investigation of Sound Speed Errors in Adaptive Beamforming. In Proceedings of the IEEE International Ultrasonics Symposium (pp. 1080-1083). IEEE.

https://doi.org/10.1109/ULTSYM.2008.0260

\section{General rights}

Copyright and moral rights for the publications made accessible in the public portal are retained by the authors and/or other copyright owners and it is a condition of accessing publications that users recognise and abide by the legal requirements associated with these rights.

- Users may download and print one copy of any publication from the public portal for the purpose of private study or research.

- You may not further distribute the material or use it for any profit-making activity or commercial gain

- You may freely distribute the URL identifying the publication in the public portal 


\title{
Investigation of Sound Speed Errors in Adaptive Beamforming
}

\author{
Iben Kraglund Holfort*, Fredrik Gran ${ }^{\dagger}$ and Jørgen Arendt Jensen* \\ ${ }^{*}$ Center for Fast Ultrasound Imaging, Department of Electrical Engineering, \\ Technical University of Denmark, DK-2800 Kgs. Lyngby, Denmark
}

${ }^{\dagger}$ GN ReSound A/S, Lautrupbjerg 9, DK-2750 Ballerup, Denmark

\begin{abstract}
Previous studies have shown that adaptive beamformers provide a significant increase of resolution and contrast, when the propagation speed is known precisely. This paper demonstrates the influence of sound speed errors on two adaptive beamformers; the Minimum Variance (MV) beamformer and the Amplitude and Phase (APES) beamformer. Simulations of a single point target are carried out in Field II, and a percentage error is applied on the speed of sound. As the error increases, $M V$ and APES provide amplitude drops of $17 \mathrm{~dB}$ and $3 \mathrm{~dB}$ on the signal strength. Two approaches to overcome this amplitude drop is proposed; diagonal loading (DL) and forward-backward (FB) averaging of the covariance matrix. The investigations show that DL provides a slightly decreased resolution and amplitude compared to FB. It is noted that APES provides more robust estimates than $\mathrm{MV}$ at the mere expense of a slight decrease of resolution. From the investigations, it is concluded the performance of the adaptive beamformers are not outperformed by the conventional delay-and-sum beamformer.
\end{abstract}

\section{INTRODUCTION}

In medical ultrasound imaging beamforming is conventionally carried out using the delay-and-sum (DS) beamformer. Thus, the achievable resolution, and contrast is dependent on the inherent compromise between the main-lobe width and the side-lobe level. Although data-dependent, adaptive beamformers have been used in other fields of array signal processing for decades, the application of adaptive beamforming to the field of medical ultrasound imaging has only recently become an area of increasing interest [1]-[4].

Adaptive beamformers are highly sensitive to steering vector errors; and robust approaches for adaptive beamforming is a widely studied subject, see e.g. [5]. In medical ultrasound imaging, the primary cause of steering vector errors is incorrect sound speed estimates. As this estimate varies with the characteristics of the different tissue types within the human body [6], the variation cannot be eliminated. In the field of medical ultrasound imaging, robust methods for adaptive beamforming have been suggested by e.g. Wang et al. [3] and Synnevåg et al. [4].

In this paper, we demonstrate the influence of sound speed errors on two adaptive beamformers; the Minimum Variance (MV) beamformer and the APES beamformer. The adaptive beamformers are implemented in the frequency domain as suggested in [7], and it provides a set of data-dependent, complex weights for each frequency sub-band. Furthermore, three different covariance matrix estimation approaches are compared; these are forward-only spatial smoothing, diagonal loading and forward-backward averaging.

\section{Sub-Band Minimum Variance Beamforming}

As in conventional beamforming, the sensor signals are presteered, so that each scan line is dynamically focused. Considering a linear array transducer with $M$ sensor elements, the $m$ th dynamically focused sensor signal along the $\ell$ th scan line is given by

$$
y_{m, \ell}(z)=s\left(\tau_{m, \ell}(z)\right) \text {, }
$$

for $m=0,1, \ldots, M-1$ and $\ell=0,1, \ldots, L-1$, where $z$ denotes the spatial position along the $\ell$ th scan line, $s(t)$ is the received waveform. The delay, $\tau_{m, \ell}(z)$, is found from the propagation path of the sound waves from the transmitting element to the focal point and returning to the receiving element, and is given by

$$
\tau_{m, \ell}(z)=\frac{\left\|\vec{r}^{(x m t)}-\vec{r}_{\ell}(z)\right\|+\left\|\vec{r}_{m}^{(r c v)}-\vec{r}_{\ell}(z)\right\|}{c},
$$

where $\vec{r}^{(x m t)}$ and $\vec{r}_{m}^{(r c v)}$ are the spatial positions of the transmitting and the $m$ th receiving sensor elements, $\vec{r}_{\ell}(z)$ denotes the focal point, and $c$ is the speed of sound.

The MV beamformer [8] is originally developed for narrowband applications. Applying MV to broad-band ultrasound data, the sensor signals are divided into sub-bands using the short-time Fourier transform. For a given point, $\vec{r}_{\ell}\left(z_{0}\right)$, the beamformer output for each spatial frequency sub-band, $k$, is given by

$$
B_{\ell}\left(k, z_{0}\right)=\mathbf{w}_{\ell}\left(k, z_{0}\right)^{H} \mathbf{Y}_{\ell}\left(k, z_{0}\right),
$$

where $\mathbf{w}_{\ell}\left(k, z_{0}\right)$ is a vector of sensor weights, $\mathbf{Y}_{\ell}\left(k, z_{0}\right)$ is a vector of short-time Fourier transforms of the segmented sensor signals, $y_{m, \ell}\left(z+z_{0}\right), z \in[-Z / 2 ; Z / 2]$. The superscripts, $\{\cdot\}^{T}$ and $\{\cdot\}^{H}$, denote the non-conjugate and the conjugate transpose, respectively. Note that the sub-band division provides the possibility of weighting both each sub-band and each point differently.

The MV beamformer continuously updates the weights, so that the variance (or power) of the beamformer output is minimized, while the response from the focus point is passed 
without distortion. The power of the beamformer output is given by

$$
\begin{aligned}
\mathcal{P}_{\ell}\left(k, z_{0}\right) & =\mathcal{E}\left\{\left|B_{\ell}\left(k, z_{0}\right)\right|^{2}\right\} \\
& =\mathbf{w}_{\ell}\left(k, z_{0}\right)^{H} \mathbf{R}_{\ell}\left(k, z_{0}\right) \mathbf{w}_{\ell}\left(k, z_{0}\right),
\end{aligned}
$$

where $\mathcal{E}\{\cdot\}$ denotes the expectation value, and $\mathbf{R}_{\ell}\left(k, z_{0}\right)$ is the covariance matrix given by

$$
\mathbf{R}_{\ell}\left(k, z_{0}\right)=\mathcal{E}\left\{\mathbf{Y}_{\ell}\left(k, z_{0}\right) \mathbf{Y}_{\ell}\left(k, z_{0}\right)^{H}\right\} .
$$

Mathematically, the MV beamformer is expressed as [8]

$$
\begin{gathered}
\min _{\mathbf{w}_{\ell}\left(k, z_{0}\right)} \mathbf{w}_{\ell}\left(k, z_{0}\right)^{H} \mathbf{R}_{\ell}\left(k, z_{0}\right) \mathbf{w}_{\ell}\left(k, z_{0}\right) \\
\quad \text { subject to } \mathbf{w}_{\ell}\left(k, z_{0}\right)^{H} \mathbf{e}\left(k, z_{0}\right)=1,
\end{gathered}
$$

where $\mathbf{e}\left(k, z_{0}\right)$ is the so-called steering vector, which characterizes the response from the focus point. The solution to the optimization problem (7) can be found in a single iteration using Lagrangian multiplier theory as [8]

$$
\mathbf{w}_{\ell}\left(k, z_{0}\right)=\frac{\mathbf{R}_{\ell}\left(k, z_{0}\right)^{-1} \mathbf{e}\left(k, z_{0}\right)}{\mathbf{e}\left(k, z_{0}\right)^{H} \mathbf{R}_{\ell}\left(k, z_{0}\right)^{-1} \mathbf{e}\left(k, z_{0}\right)},
$$

provided that $\mathbf{R}_{\ell}\left(k, z_{0}\right)^{-1}$ exists. Due to presteering and subband division, the response from the focus point will resemble a plane wave incident directly onto the array. Thus, the steering vector is constant across the array and independent on the frequency, and it simply becomes a $M \times 1$-vector of ones.

\section{A. Spatial Smoothing}

To obtain a useful estimate of the covariance matrix, a number of realizations is needed. Here, data from a single acquisition is divided into a number of subgroups and averaged spatially, as suggested in [9]. The spatially smoothed covariance matrix estimate is obtained by dividing the array into $P$ overlapping subarrays of length $L$. For each subarray, a sub-covariance matrix is estimated, and these are averaged across the array. The covariance matrix estimate can be expressed as

$$
\hat{\mathbf{R}}=\frac{1}{P} \sum_{p=0}^{P-1} \mathbf{G}_{p} \mathbf{G}_{p}^{H},
$$

where $\mathbf{G}_{p}$ denotes the $p$ th subarray given by

$$
\mathbf{G}_{p}=\left[\begin{array}{llll}
Y_{p}(\omega) & Y_{p+1}(\omega) & \ldots & Y_{p+L-1}(\omega)
\end{array}\right]^{T},
$$

for $p=0,1, \ldots, P-1$. Note that this reduces the dimension of the covariance matrix, and thus the number of weights will be reduced correspondingly. The reduced weight vector, $\widetilde{\mathbf{w}}$, is applied to the data by averaging over the $P$ subarrays, which is expressed as

$$
B\left(\vec{r}_{p}\right)=\tilde{\mathbf{w}}^{H} \frac{1}{P} \sum_{p=0}^{P-1} \mathbf{G}_{p} .
$$

\section{B. APES Beamforming}

The APES (Amplitude and Phase Estimation) Beamformer is based on the analogue spectral estimator [10]. The APES estimate can be found by replacing the covariance matrix in (8) by

$$
\mathbf{Q}=\hat{\mathbf{R}}-\hat{\mathbf{G}} \hat{\mathbf{G}}^{H}, \quad \hat{\mathbf{G}}=\frac{1}{P} \sum_{p=0}^{P-1} \mathbf{G}_{p}
$$

with $\hat{\mathbf{R}}$ and $\mathbf{G}_{p}$ given in (9)-(10). For more on the APES algorithm see e.g. [11].

\section{Diagonal Loading}

A commonly used approach for robust adaptive beamforming is to use diagonal loading [5], where a small scalar value, $\varepsilon$, is added to the diagonal of the covariance matrix estimate.

$$
\hat{\mathbf{R}}_{\varepsilon}=\hat{\mathbf{R}}+\varepsilon \mathbf{I},
$$

where $I$ denotes the identity matrix. The choice of $\varepsilon$ will have a large influence on the provided weights. The larger $\varepsilon$ becomes, the more the diagonal will dominate the result; and as shown in e.g. [4], the result will converge to the DS result. As suggested in [4], $\varepsilon$ is chosen to be relative to the trace, $\operatorname{Tr}\{\cdot\}$, of $\hat{\mathbf{R}}$. In Sec. III, a value of $\varepsilon=10^{-3} \operatorname{Tr}\{\hat{\mathbf{R}}\}$ is used.

\section{Forward-Backward Averaging}

The covariance matrix estimate in (9) is sometimes referred to as the forward-only estimate. Yet another covariance matrix estimate is the Forward-Backward (FB) average [11] defined by

$$
\tilde{\mathbf{R}}=\frac{1}{2}\left(\hat{\mathbf{R}}+\mathbf{J} \hat{\mathbf{R}}^{T} \mathbf{J}\right), \quad \mathbf{J}=\left[\begin{array}{lll}
0 & & 1 \\
& . & \\
1 & & 0
\end{array}\right] .
$$

This approach has previously been used in radar imaging in combination with APES by Wang et al. [12].

\section{Application to Ultrasound Data}

The two adaptive beamformers are applied on simulated ultrasound data, obtained using Field II [13], [14]. For the simulations, a $7 \mathrm{MHz}$ linear array transducer with $\lambda / 2$ spacing was used. Data is obtained using a single element as the transmitting aperture and all $M=128$ elements as the receiving aperture. The MV and APES beamformers are implemented in the frequency domain using the shorttime Fourier transform with a segment size corresponding to the length of the excitation pulse convolved with the twoway impulse response of the transducer. A subarray size of $L=\frac{M}{4}=32$ was used, and before beamforming, additional white, Gaussian noise was added to each of the sensor signals. The signal-to-noise ratio was $60 \mathrm{~dB}$, and the correct sound speed was $1540 \mathrm{~m} / \mathrm{s}$.

The sound speed estimate is changed percentage-wise from the true value by $2 \%, 4 \%$, and $6 \%$, which correspond to velocities of $\{1571,1602,1632\} \mathrm{m} / \mathrm{s}$. The adaptive beamformers with the three covariance matrix estimation approaches are 

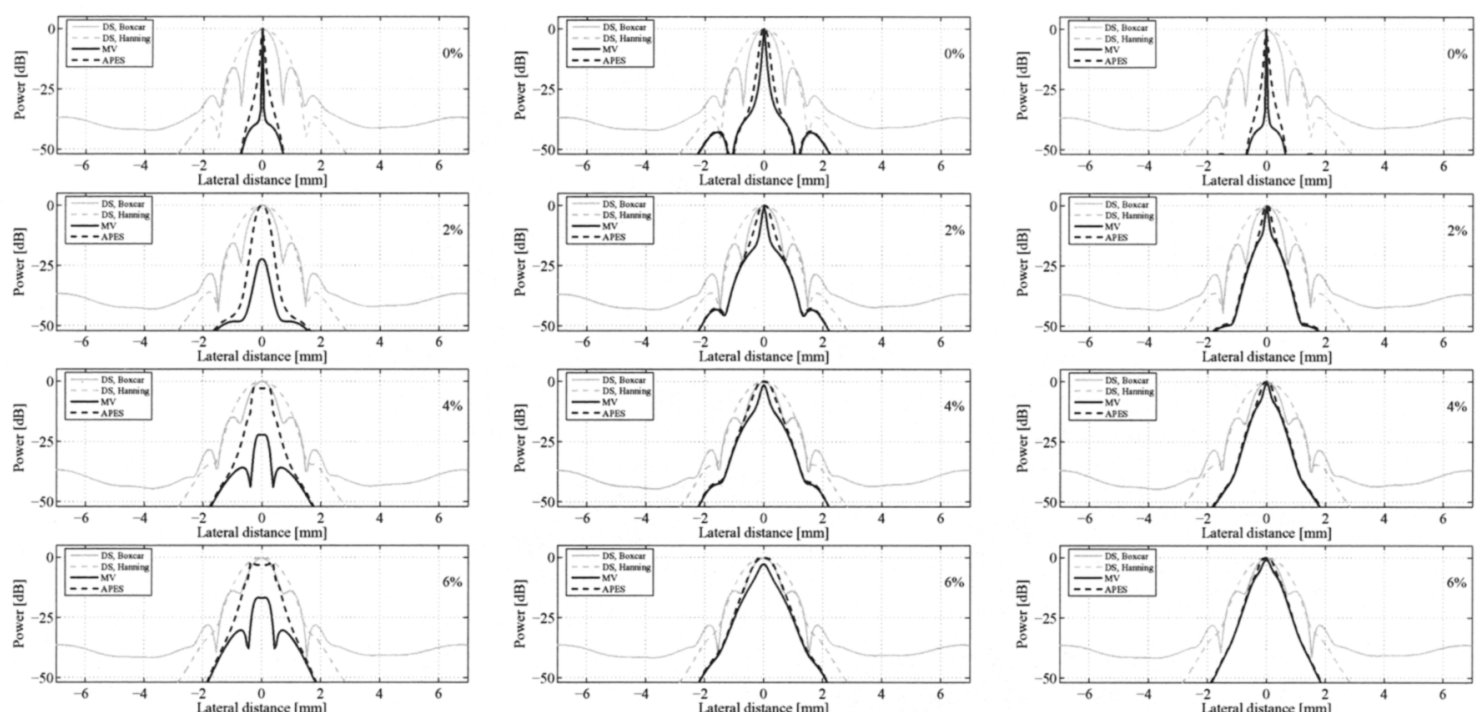

(a) Forward-only

(b) Diagonal loading

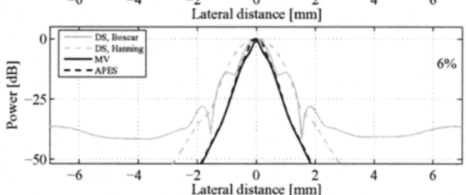

(c) Forward-Backward

Fig. 1. Lateral variation of the beamformed responses at depth $z=40 \mathrm{~mm}$. The beamformed responses are DS, Boxcar (gray solid), DS, Hanning (gray dashed), MV (black solid) and APES (black dashed). (a) Forward-only, no diagonal loading has been applied. (b) Forward-only, diagonal loading factor of $\varepsilon=10^{-3} \operatorname{Tr}\{\mathbf{R}\}$ is used. (c) Forward-backward averaging is used (no diagonal loading). The percentage error of the sound speed is given at the top right in each figure.
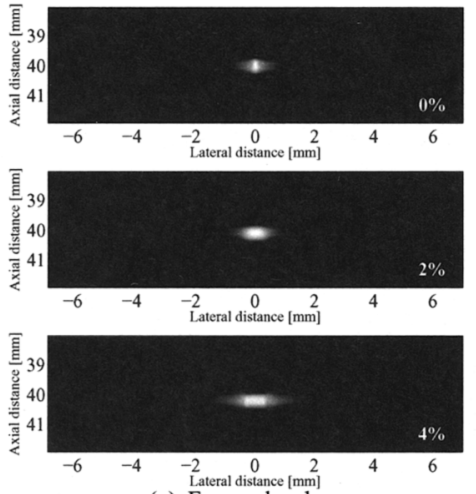

(a) Forward-only
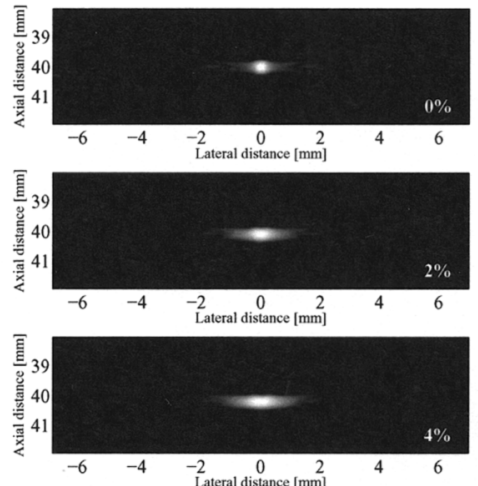

(b) Diagonal loading
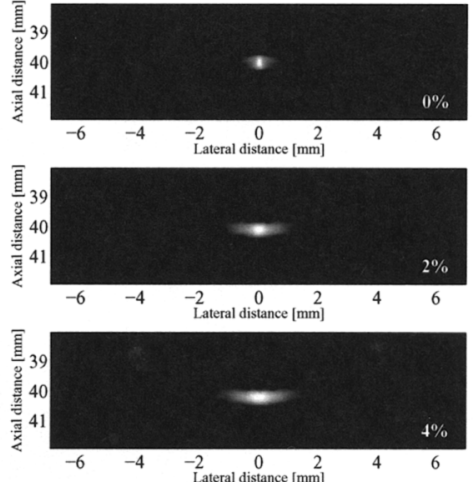

(c) Forward-Backward

Fig. 2. Minimum variance beamformed responses from a single point target for different percentage error on the sound speed. Images are shown with a dynamic range of $50 \mathrm{~dB}$.
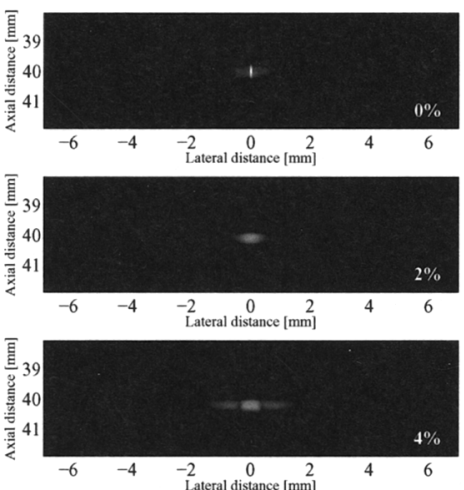

(a) Forward-only
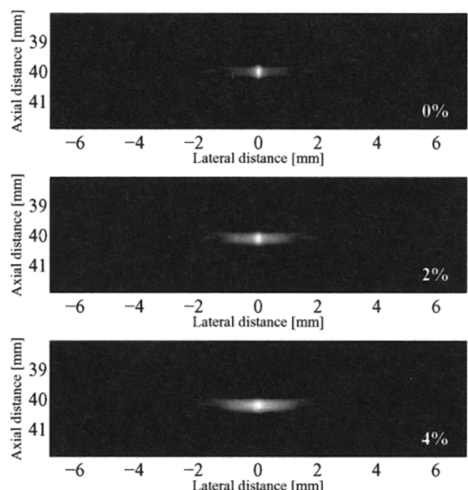

(b) Diagonal loading
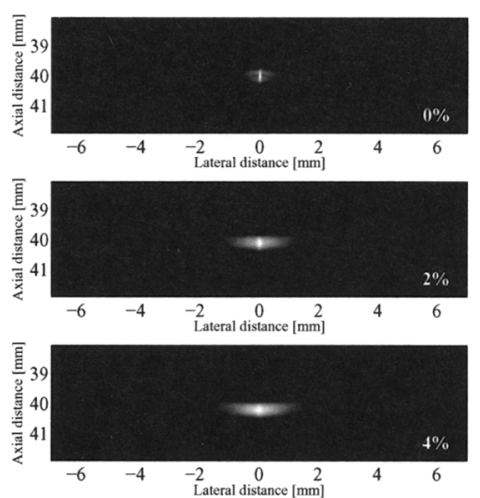

(c) Forward-Backward

Fig. 3. APES beamformed responses from a single point target for different percentage error on the sound speed. Images are shown with a dynamic range of $50 \mathrm{~dB}$. 

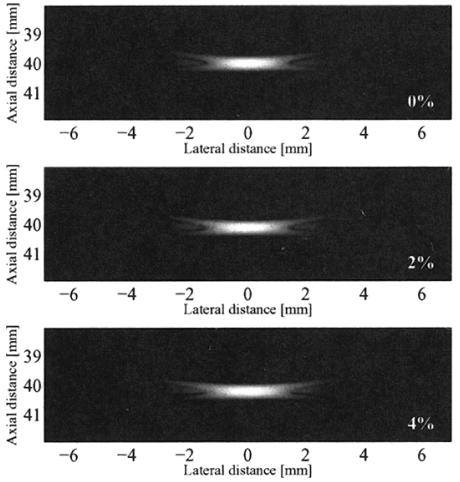

Fig. 4. Delay-and-sum (Hanning weights) beamformed responses from a single point target for different percentage error on the sound speed. Images are shown with a dynamic range of $50 \mathrm{~dB}$.

compared to the DS beamformer using Boxcar and Hanning weights. The lateral variation of the beamformed responses os a single point target are seen in Fig. 1. Furthermore, a selection of the MV and APES beamformed responses are seen in Fig. 2 and Fig. 3. For comparison the DS beamformed responses using Hanning weights are shown in Fig. 4.

At the top in Fig. 1(a)-(c), the beamformed responses for the correct sound speed are shown. The inherent compromise between main-lobe width and side-lobe level is evident from these figures; the Hanning weights lower the side-lobe level at the expense of a broader main-lobe compared to the Boxcar weights.

It is seen that the MV and APES beamformers provide a significant increase in both resolution and contrast. Furthermore, it is noted that diagonal loading results in a slightly decreased resolution; as would be expected due to the convergence to the DS solution. As seen the forward-backward (FB) approach does not diminish the resolution significantly. However, these investigations are based on single point targets; as adaptive beamformers are strictly data-dependent, a truly thorough investigation should include a variety of simulations.

As the percentage error increases, the MV and APES beamformers are not as robust compared to the DS beamformer. Using the forward-only approach without diagonal loading, MV and APES provide amplitude drops on the signal strength of $17 \mathrm{~dB}$ and $3 \mathrm{~dB}$, respectively. This amplitude drop can be avoided by using either diagonal loading or FB as seen in Fig. 1(b)-(c). However, a slight amplitude drop is seen for $\mathrm{MV}$ at $4 \%$ and $6 \%$ error using diagonal loading.

In general, it is observed that the APES beamformer provide more robust estimates than $\mathrm{MV}$; in terms of less amplitude drop at the expense of a slightly decreased resolution. However, the resolution decrement seems insignificant compared to the robustness gained from using this beamformer.

The investigations have shown that the performance of the adaptive beamformers are significantly decreased for increasing sound speed errors. However, it is noted that the conventional DS beamformer does not outperform the adaptive beamformers within the investigated region of errors. Thus, incorrect sound speed sound estimates will not lead to a performance degradation worse than that of the conventional DS beamformer.

\section{CONCLUSIONS}

The adaptive MV and APES beamformers have been applied to simulated ultrasound data with three different covariance matrix estimation approaches; forward-only spatial smoothing, diagonal loading (DL), and forward-backward (FB) averaging. For correct speed of sound estimates, both MV and APES provide significant performance gains with respect to resolution and contrast. As the sound speed error increases, MV and APES provide $17 \mathrm{~dB}$ and $3 \mathrm{~dB}$ amplitude drops on the signal strength. However, using DL or FB the amplitude drop is avoided. Furthermore, it is seen that APES provides more robust estimates than MV. In general, it is observed that even though the performance of the adaptive beamformers is much degraded, when incorrect speed of sound is used in the beamforming. However, the performance was shown to outperform the conventional delay-and-sum beamformer.

\section{ACKNOWLEDGMENT}

A thanks goes to Prof. Sverre Holm, Dr. Andreas Austeng and PhD Students Johan-Fredrik Synnevåg and Ann Blomberg for valuable input. They are all with the University of Oslo, Norway. This work was supported by grant 26-04-0024 from the Danish Science Foundation and by B-K Medical ApS, Denmark.

\section{REFERENCES}

[1] J. A. Mann and W. F. Walker, "A Constrained Adaptive Beamformer for Medical Ultrasound: Initial Results," in Proc. IEEE Ultrason. Symp., Oct. 2002, vol. 2, pp. 1807-1810.

[2] M. Sasso and C. Cohen-Bacrie, "Medical Ultrasound Imaging Using the Fully Adaptive Beamformer," in Proc. IEEE Int. Conf. Acous., Speech, Sig. Pro., March 2005, vol. 2, pp. 489-492.

[3] Z. Wang, J. Li, and R. Wu, "Time-Delay- and Time-Reversal-Based Robust Capon Beamformers for Ultrasound Imaging," IEEE Trans. Med. Imag., vol. 24, no. 10, pp. 130-1322, Oct. 2007.

[4] J.-F. Synnevåg, A. Austeng, and S. Holm, "Adaptive Beamforming Applied to Medical Ultrasound Imaging," IEEE Trans. Ultrason., Ferroelec., Freq. Contr., vol. 54, no. 8, pp. 1606-1613, Aug. 2007.

[5] J. Li and P. Stoica, Robust Adaptive Beamforming, John Wiley \& Sons, New York, 2006.

[6] S. A. Goss, R. L. Johnston, and F. Dunn, "Comprehensive compilation of empirical ultrasonic properties of mammalian tissues," J. Acoust. Soc. Am., vol. 64, pp. 423-457, 1978.

[7] I. K. Holfort, F. Gran, and J. A. Jensen, "Minimum Variance Beamforming for High Frame-Rate Ultrasound Imaging," in Proc. IEEE Ultrason. Symp., Oct. 2007, pp. 1541-1544.

[8] J. Capon, "High-Resolution Frequency-Wavenumber Spectrum Analysis," Proc. IEEE, vol. 57, no. 8, pp. 1408-1418, August 1969.

[9] T.-J. Shan and T. Kailath, "Adaptive Beamforming for Coherent Signals and Interference," IEEE Trans. Acous., Speech, Sig. Pro., vol. 33, no. 3, pp. 527-536, June 1985.

[10] J. Li and P. Stoica, "An Adaptive Filtering Approach to Spectral Estimation and SAR Imaging," IEEE Trans. Sig. Pro., vol. 44, pp. 1469-1484, 1996.

[11] P. Stoica and R. Moses, Spectral Analysis of Signals, Pearson Prentice Hall, New Jersey, 2005.

[12] Y. Wang, X. Li, Y. Sun, J. Li, and P. Stoica, "Adaptive Imaging for Forward-Looking Ground Penetrating Radar," IEEE Trans. Aero. Electron. Sys., vol. 41, pp. 922-936, 2005.

[13] J. A. Jensen and N. B. Svendsen, "Calculation of pressure fields from arbitrarily shaped, apodized, and excited ultrasound transducers," IEEE Trans. Ultrason., Ferroelec., Freq. Contr., vol. 39, pp. 262-267, 1992.

[14] J. A. Jensen, "Field: A program for simulating ultrasound systems," Med. Biol. Eng. Comp., vol. 10th Nordic-Baltic Conference on Biomedical Imaging, Vol. 4, Supplement 1, Part 1, pp. 351-353, $1996 \mathrm{~b}$. 